\title{
Reproducibility of MRI Dixon-Based Attenuation Correction in Combined PET/MR with Applications for Lean Body Mass Estimation
}

\author{
Ivo Rausch ${ }^{1}$, Petra Rust ${ }^{2}$, Matthew D. DiFranco ${ }^{1}$, Martin Lassen ${ }^{1}$, Andreas Stadlbauer ${ }^{3}$, Marius E. Mayerhoefer ${ }^{3}$, \\ Markus Hartenbach ${ }^{4}$, Marcus Hacker ${ }^{4}$, and Thomas Beyer ${ }^{1}$ \\ ${ }^{I}$ Center for Medical Physics and Biomedical Engineering, Medical University of Vienna, Vienna, Austria; ${ }^{2}$ Department of Nutritional \\ Sciences, University of Vienna, Vienna, Austria; ${ }^{3}$ Division of General and Pediatric Radiology, Department of Biomedical Imaging \\ and Image-Guided Therapy, Medical University of Vienna, Vienna, Austria; and ${ }^{4}$ Division of Nuclear Medicine, Department of \\ Biomedical Imaging and Image-guided Therapy, Medical University of Vienna, Vienna, Austria
}

The aim of this study was to assess the reproducibility of standard, Dixon-based attenuation correction (MR-AC) in PET/MR imaging. A further aim was to estimate a patient-specific lean body mass (LBM) from these MR-AC data. Methods: Ten subjects were positioned in a fully integrated PET/MR system, and 3 consecutive multibed acquisitions of the standard MR-AC image data were acquired. For each subject and MR-AC map, the following compartmental volumes were calculated: total body, soft tissue (ST), fat, lung, and intermediate tissue (IT). Intrasubject differences in the total body and subcompartmental volumes (ST, fat, lung, and IT) were assessed by means of coefficients of variation (CVs) calculated across the 3 consecutive measurements and, again, across these measurements but excluding those affected by major artifacts. All subjects underwent a body composition measurement using air displacement plethysmography (ADP) that was used to calculate a reference $L_{B M} M_{A D P}$. A second $L B M$ estimate was derived from available MR-AC data using a formula incorporating the respective tissue volumes and densities as well as the subject-specific body weights. A third LBM estimate was obtained from a sex-specific formula ( $\left(\mathrm{LBM}_{\mathrm{Formula}}\right)$. Pearson correlation was calculated for $\mathrm{LBM}_{\mathrm{ADP}}$, $\mathrm{LBM}_{\mathrm{MR}-\mathrm{AC}}$, and $\mathrm{LBM}_{\mathrm{Formula}}$. Further, linear regression analysis was performed on $\mathrm{LBM}_{\mathrm{MR}-\mathrm{AC}}$ and $\mathrm{LBM}_{\mathrm{ADP}}$. Results: The mean CV for all 30 scans was $2.1 \pm 1.9 \%$ (TB). When missing tissue artifacts were excluded, the CV was reduced to $0.3 \pm 0.2 \%$. The mean CVs for the subcompartments before and after exclusion of artifacts were $0.9 \pm$ $1.1 \%$ and $0.7 \pm 0.7 \%$ for the ST, $2.9 \pm 4.1 \%$ and $1.3 \pm 1.0 \%$ for fat, and $3.6 \pm 3.9 \%$ and $1.3 \pm 0.7 \%$ for the IT, respectively. Correlation was highest for $\mathrm{LBM}_{\mathrm{MR}-\mathrm{AC}}$ and $\mathrm{LBM}_{\mathrm{ADP}}(r=0.99)$. Linear regression of data excluding artifacts resulted in a scaling factor of 1.06 for $\mathrm{LBM}_{\mathrm{MR}-\mathrm{AC}}$. Conclusion: $\mathrm{LBM}_{\mathrm{MR}-\mathrm{AC}}$ is shown to correlate well with standard LBM measurements and thus offers routine LBM-based SUV quantification in PET/MR. However, MR-AC images must be controlled for systematic artifacts, including missing tissue and tissue swaps. Efforts to minimize these artifacts could help improve the reproducibility of MR-AC.

\footnotetext{
Received Oct. 20, 2015; revision accepted Feb. 8, 2016.

For correspondence or reprints contact: Ivo Rausch, Quantitative Imaging and Medical Physics Group (QIMP), Center for Medical Physics and Biomedical Engineering, Medical University of Vienna, Waehringer Guertel 18-20, 1090 Vienna, Austria.

E-mail: ivo.rausch@meduniwien.ac.at

Published online Feb. 25, 2016.

COPYRIGHT (C 2016 by the Society of Nuclear Medicine and Molecular Imaging, Inc.
}

Key Words: PET/MR; Dixon-based MR-AC reproducibility; lean body mass estimation; body composition measurement

J Nucl Med 2016; 57:1096-1101

DOI: 10.2967/jnumed.115.168294

D ual-modality PET/CT imaging systems, combining CT with PET into a single imaging system, have become standard for acquiring colocalized metabolic and anatomic information in clinical practice (1). The effectiveness of PET/CT is due, in part, to the complementary role of $\mathrm{CT}$ in providing both an anatomic framework and reliable, quantitative attenuation values for tissues inside the field of view of the PET for CT-based attenuation correction (2). After the introduction of dual-modality MR and PET imaging (PET/MR), novel clinical applications of hybrid imaging are being actively developed (3). PET/MR imaging has the advantage of offering superior soft-tissue (ST) contrast and a wide array of functional, morphologic, and even metabolic clinical imaging protocols (4) within a single examination. However, correct attenuation correction is a prerequisite for the quantitative assessment of PET images, but the use of MR image information in PET/MR imaging for deriving MR-based attenuation correction (MR-AC) is still a matter of debate (5).

Furthermore, the reproducibility of quantitative PET results is of critical importance, particularly in the context of intrasubject therapy response assessment. The reproducibility of PET data is closely related to the reproducibility of the implemented attenuationcorrection method. In the context of PET/MR, this is a matter of debate because the current implementations of MR-AC are known to be affected by image artifacts ( 6 ). Several of these artifacts and their effect on MR-AC have been discussed, such as truncation due to a limited field of view or tissue swap effects in Dixon-based MR-AC maps $(7,8)$, thus limiting the reproducibility of MR-AC and subsequent attenuation-corrected PET data (9).

Despite the general drawbacks of MR-AC methods, the Dixonbased MR-AC provides information on the amount and distribution of adipose tissue present in each subject undergoing a PET/MR imaging examination. This was already shown for Dixon-type images by Wald et al. (10) and Ludwig et al. (11) and confirmed in a study by Jochimsen et al. (12). Hence, standard MR-AC algorithms generate 
TABLE 1

Summary of Results for All Subjects

\begin{tabular}{|c|c|c|c|c|c|c|c|c|}
\hline Subject & Sex & Height $(\mathrm{cm})$ & $\begin{array}{c}\text { Body } \\
\text { weight }(\mathrm{kg})\end{array}$ & $\mathrm{Fat}_{\mathrm{ADP}}(\mathrm{kg})$ & $\mathrm{LBM}_{\mathrm{ADP}}(\mathrm{kg})$ & LBM $_{\text {Formula }}(\mathrm{kg})$ & $\begin{array}{c}\text { LBM }_{\text {MR-AC }} \text { (all MR-AC } \\
\text { maps/excluding artifacts) (kg) }\end{array}$ & $\begin{array}{c}\text { Scaled } \\
\text { LBM }_{\text {MR-AC }}(\mathrm{kg})\end{array}$ \\
\hline 1 & $\mathrm{~F}$ & 169 & 56.4 & 11.9 & 44.5 & 43.9 & $42.9 / 42.8$ & 45.4 \\
\hline 2 & M & 177 & 82.5 & 22.2 & 60.3 & 63.0 & $55.9 / 55.9$ & 59.2 \\
\hline 3 & M & 178 & 67.4 & 14.2 & 53.3 & 55.8 & $51.9 / 51.9$ & 55.0 \\
\hline 4 & M & 169 & 71.4 & 16.5 & 54.9 & 55.7 & $52.0 / 51.8$ & 54.9 \\
\hline 5 & $\mathrm{~F}$ & 170 & 59.0 & 16.4 & 42.7 & 45.3 & $40.6 / 40.5$ & 43.0 \\
\hline 6 & $F$ & 167 & 56.0 & 12.6 & 43.4 & 43.3 & $40.6 / 40.3$ & 42.7 \\
\hline 7 & $\mathrm{~F}$ & 160 & 56.8 & 15.5 & 41.3 & 42.1 & $38.5 / 38.5$ & 40.8 \\
\hline 8 & M & 180 & 79.0 & 5.6 & 73.4 & 62.3 & $63.9 /-\left(67.1^{\star}\right)$ & $-\left(71.1^{\star}\right)$ \\
\hline 9 & M & 179 & 71.7 & 5.0 & 66.8 & 58.3 & $59.3 /-\left(60.7^{\star}\right)$ & $-\left(64.4^{\star}\right)$ \\
\hline 10 & $\mathrm{~F}$ & 169 & 56.9 & 5.8 & 51.2 & 44.1 & $47.6 / 48.4$ & 51.3 \\
\hline
\end{tabular}

${ }^{\star}$ For these subjects, tissue swaps occurred in all repeated scans. Values in parentheses are extracted from 1 scan each for which tissue swaps were corrected manually. This was done by inverting ST and fat values in MR-AC maps in respective regions. This is predictive for LBM with no tissue swaps but is not supposed to result in same LBM gained from artifact-free MR-AC image.

LBM $_{M R-A C}$ column shows values of LBM gained for all acquisitions with and without tissue swap and missing tissue artifacts. Scaled $\mathrm{LBM}_{\mathrm{MR}-\mathrm{AC}}$ column shows values scaled by scaling factor gained from linear regression.

image information that can be used to estimate a patient-specific lean body mass (LBM) for potentially improved quantification of PET data in PET/MR imaging (11).

In practice, a PET study is performed as a static measurement over a fixed amount of time (e.g., 5 min). Unfortunately, an absolute quantification of the metabolic activity is not possible a priori. The uptake of labeled tracer depends on the amount of available tracer and its ratio to the unlabeled analog (13). Kinetic modeling based on dynamic acquisitions, continuous measurement of the blood activity, and extensive postprocessing can be used to overcome this limitation, but such protocols are not accepted in clinical routine. Instead, the concept of the SUV is used widely $(14,15)$. SUV relies on the normalization of the PET signal with an estimate of tracer availability based on the injected total amount of tracer activity and a patient-specific measure, such as body weight $\left(\mathrm{SUV}_{\mathrm{bw}}\right)$, body surface area $\left(\mathrm{SUV}_{\mathrm{BSA}}\right)$, or LBM $\left(\mathrm{SUV}_{\mathrm{LBM}}\right)(16)$. $\mathrm{SUV}_{\mathrm{LBM}}$ has been suggested as an estimate of the true metabolic rates that is better than $\operatorname{SUV}_{\mathrm{bw}}(15,16)$. Subsequently, several LBM formulas based on patient weight and height, body surface area, and sex-specific correction factors have been proposed, without a single formula-based estimation being in widespread clinical use today (16-18).

Therefore, the aim of this PET/MR-specific study was 2-fold: first, to assess the reproducibility of standard Dixon-based MR-AC, and second, to assess the usefulness of Dixon-based MR-AC for an accurate and clinically viable estimation of patient-specific LBM.

\section{MATERIALS AND METHODS}

\section{Subjects}

Ten subjects ( 5 men, 5 women) were included in this study. All volunteers were selected from a separate study cohort in which subjects underwent a body composition measurement using air displacement plethysmography (ADP) (19) at the Institute of Nutritional Science at the University of Vienna. ADP is a technique to measure the body volume, which is used together with the weight of the subject to calculate the body composition based on multicompartment models $(19,20)$. ADP measurements were performed using a BOD POD system (BodPod; COSMED USA, Inc.) with the subjects dressed in light clothing. Prior to the ADP measurements, the ADP subjects were asked to void their bladders. All subjects were weighed on an accurate digital scale and seated in the chamber of the BOD POD system. Body volume was measured twice for $40 \mathrm{~s}$ each. Appropriate corrections for thoracic gas volume and skin surface area artifacts in adults were applied to the raw measurement before the actual body volume of each subject was calculated. Body density values were obtained from the measured body weight and calculated body volume. The percentage fat and $\mathrm{LBM}_{\mathrm{ADP}}$ were obtained from body density using Siri's equation (20).

The average age of the subjects was $32 \pm 7 \mathrm{y}$. The mean body weight was $66 \pm 10 \mathrm{~kg}$, and the mean height was $172 \pm 6 \mathrm{~cm}$, yielding an average body mass index of $22 \pm 2 \mathrm{~kg} / \mathrm{m}^{2}$. The fat fraction assessed with ADP ranged from $7 \%$ to $28 \%$. Detailed information for each volunteer is given in Table 1 .

The study was approved by the local ethics committee, and participating subjects gave their written informed consent to participate in this study.

\section{Acquisitions}

All subjects underwent total-body, standard Dixon-based MR imaging to generate total-body, MR-based attenuation maps (21) within $3 \mathrm{~d}$ of the ADP measurement. MR imaging was performed on a Biograph mMR PET/MR system (Siemens Healthcare) running on software version VB20.

For the acquisition of the total-body MR-AC maps, subjects were positioned head-first-supine on the PET/MR patient table to replicate positioning in a clinical total-body acquisition scenario. Similar to the clinical standard, the head-and-neck coil in combination with all available $(n=4)$ flexible body surface coils was used to cover an imaging range from the neck to the knees. Three successive Dixonbased sequences were acquired without repositioning the subjects in between scans, covering an axial imaging range from head to toe for deriving total-body MR-AC data. The following standard settings were used for the MR-AC sequence: repetition time, $3.6 \mathrm{~ms}$; echo time 1, $1.23 \mathrm{~ms}$; echo time $2,2.46 \mathrm{~ms}$; and flip angle, $10^{\circ}$. For the acquisition of 


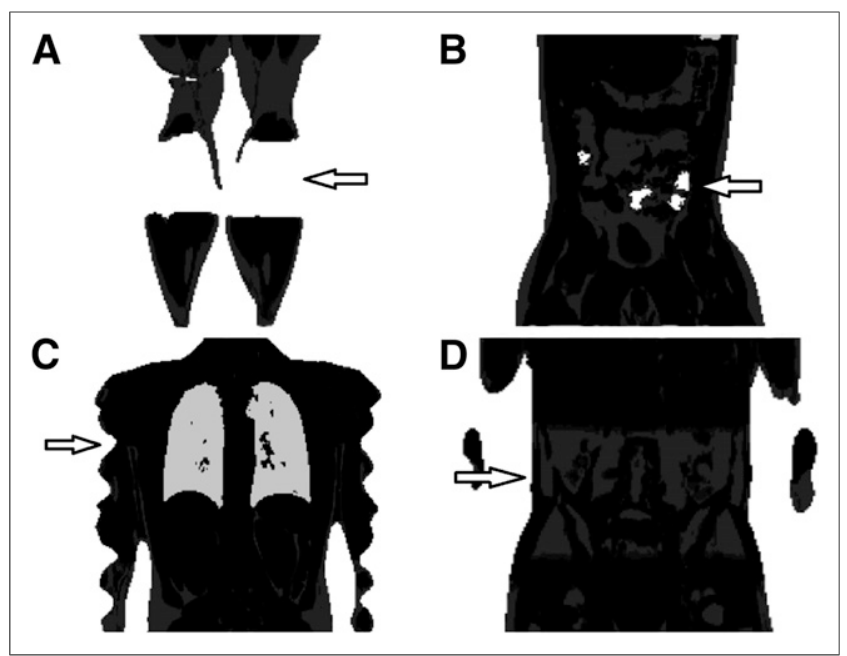

FIGURE 1. Examples of typical image artifacts in Dixon-based attenuation maps: missing tissue artifact (A), air cavities (B), truncation artifacts $(C)$, and tissue swap artifact (D) with inverted ST and fat distributions in central bed position.

relevant data of the thorax and upper abdomen, subjects were instructed to hold their breath in full-inspiration. All other acquisitions were performed in free-breathing, resulting in 3 composed total-body Dixonbased MR-AC maps per subject.

\section{Reproducibility}

An experienced imaging physicist inspected all MR-AC maps visually for artifacts. Noticeable artifacts were categorized into 4 categories: truncation, tissue swaps, air cavities, and missing tissue (Fig. 1).

Compartment volumes were calculated for all MR-AC maps using an in-house script (MATLAB version R2013b; The MathWorks). For each tissue compartment, the total volume was estimated from the number of voxels with the respective values multiplied by the volume of a single voxel. The voxel values of the compartments correspond to the linear attenuation coefficient $(1 / \mathrm{cm})$ multiplied by a factor of $10^{4}$. Voxel values were 1,000 (ST), 854 (fat), 927 (intermediate tissue [IT]), and 224 (lungs). IT describes a class of voxels that consists of a balanced mix of $50 \pm 5 \%$ of fat and ST and, therefore, is assigned the average attenuation value of fat and water. The total-body volume was calculated for each MR-AC map by adding all the compartment volumes.

For all subjects, coefficients of variation $(\mathrm{CVs})$ of the total-body volume were calculated across the 3 consecutive measurements. CV calculations were repeated for all measurements but after removal of the lung compartment to exclude effects caused by different respiratory states between the measurements. Further, $\mathrm{CV}$ calculations were repeated for all measurements but after exclusion of measurements with missing tissue artifacts without and with the lung compartment excluded.

Furthermore, $\mathrm{CV}$ of the subcompartment volumes relative to the total-body volume were calculated across the 3 consecutive measurements before and after exclusion of acquisitions with tissue swaps and missing tissue artifacts. The motivation of excluding acquisitions with missing tissue and tissue swap artifacts was to estimate the reproducibility for standard acquisitions without major image distortions considering that several solutions exist to mitigate the effects of truncation, tissue swaps, and susceptibility effects (3).

\section{LBM Estimation}

For all subjects, LBM was estimated in 3 variants: from standard MR-AC ( $\left.\mathrm{LBM}_{\mathrm{MR}-\mathrm{AC}}\right)$, from a sex-specific formula ( $\left.\mathrm{LBM}_{\mathrm{Formula}}\right)$, and from ADP measurements $\left(\mathrm{LBM}_{\mathrm{ADP}}\right)$. The calculation of the subjectspecific $\mathrm{LBM}_{\mathrm{MR}-\mathrm{AC}}$ was based on:

$$
\mathrm{LBM}_{\mathrm{MR}-\mathrm{AC}}=\mathrm{m}_{\text {subject }} \frac{\left(\mathrm{V}_{\mathrm{ST}} \times \rho_{\mathrm{ST}}\right)+\left(\mathrm{V}_{\mathrm{IT}} \times \rho_{\mathrm{IT}} \times 0.5\right)}{\left(\mathrm{V}_{\mathrm{ST}} \times \rho_{\mathrm{ST}}\right)+\left(\mathrm{V}_{\mathrm{fat}} \times \rho_{\mathrm{fat}}\right)+\left(\mathrm{V}_{\mathrm{IT}} \times \rho_{\mathrm{IT}}\right)},
$$

where $\mathrm{m}$ is the total-body mass in $(\mathrm{kg}), \mathrm{V}$ are the respective compartment volumes in (L), and $\rho$ are the tissue-dependent densities $\left(\rho_{\mathrm{ST}}=1.1 \mathrm{~kg} / \mathrm{L}, \rho_{\mathrm{F}}=0.9 \mathrm{~kg} / \mathrm{L}, \rho_{\mathrm{IT}}=1 \mathrm{~kg} / \mathrm{L}\right)(22) . \mathrm{LBM}_{\text {MR-AC }}$ was calculated in 2 ways: first, with the average value of all compartment volumes for the 3 acquisitions, and second, after exclusion of scans with tissue swaps and missing tissue artifacts, as these type of image distortions are suspected to affect the CV most significantly.

$\mathrm{LBM}_{\text {Formula }}$ was calculated for men and women using the approach described by Green and Duffull (23):

$$
\begin{gathered}
\text { Men: } \operatorname{LBM}_{\text {Formula }}=1.1 \times \mathrm{m}_{\text {subject }}-128 \times\left(\frac{\mathrm{m}_{\text {subject }}}{\mathrm{h}_{\text {subject }}}\right)^{2} \\
\text { Women: } \operatorname{LBM}_{\text {Formula }}=1.07 \times \mathrm{m}_{\text {subject }}-148 \times\left(\frac{\mathrm{m}_{\text {subject }}}{\mathrm{h}_{\text {subject }}}\right)^{2},
\end{gathered}
$$

where $h_{\text {subject }}$ is the subject height in $\mathrm{cm}$, and $\mathrm{m}_{\text {subject }}$ is the subject weight in $\mathrm{kg}$.

$\mathrm{LBM}_{\mathrm{ADP}}$ was considered the gold standard. The correlation between $\mathrm{LBM}_{\mathrm{ADP}}, \mathrm{LBM}_{\mathrm{MR}-\mathrm{AC}}$, and $\mathrm{LBM}_{\mathrm{Formula}}$ was assessed using Pearson correlation coefficient together with the corresponding $95 \%$ confidence intervals (CIs) and tested for correlation (null hypothesis: correlation $=0$ ). A linear regression was used to derive a scaling factor between $\mathrm{LBM}_{\mathrm{MR}-\mathrm{AC}}$ and $\mathrm{LBM}_{\mathrm{ADP}}$. This factor was then used to scale $\mathrm{LBM}_{\mathrm{MR}-\mathrm{AC}}$ estimates extracted from MR-AC maps that did not contain tissue swap or missing tissue artifacts to $\mathrm{LBM}_{\mathrm{ADP}}$

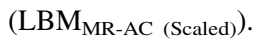

\section{RESULTS}

\section{Reproducibility}

Artifacts. Truncation artifacts were present in all scans of all subjects. For each subject, air cavities were present in at least 1 of 3 MR-AC maps. Missing tissue artifacts and tissue swaps were observed in scans of 4 subjects each. Tissue swaps were recognized in 2 subjects in all repeated scans. Figure 1 and Table 2 summarize all relevant findings.

CVs. The average CV of the total-body volume for all scans before and after exclusion of lung tissue classes was $2.1 \pm 1.9 \%$ and $2.3 \pm 2.0 \%$, respectively. After exclusion of the scans with missing tissue artifacts, the average $\mathrm{CV}$ for the total-body volume was $0.3 \pm 0.2 \%$ and $0.3 \pm 0.3 \%$ before and after the exclusion of

TABLE 2

Artifacts in Repeated Scans

\begin{tabular}{lcccc}
\hline Parameter & $\begin{array}{c}\text { Missing } \\
\text { tissue }\end{array}$ & $\begin{array}{c}\text { Air } \\
\text { cavities }\end{array}$ & Truncation & $\begin{array}{c}\text { Tissue } \\
\text { swaps }\end{array}$ \\
\hline Subjects & 4 of 10 & 10 of 10 & 10 of 10 & 4 of 10 \\
Scans & 6 of 30 & 28 of 30 & 30 of 30 & 8 of 30
\end{tabular}

Subjects row gives number of subjects in whom respective artifacts were observed. Scans row gives number of scans in total in which respective artifacts were observed. 


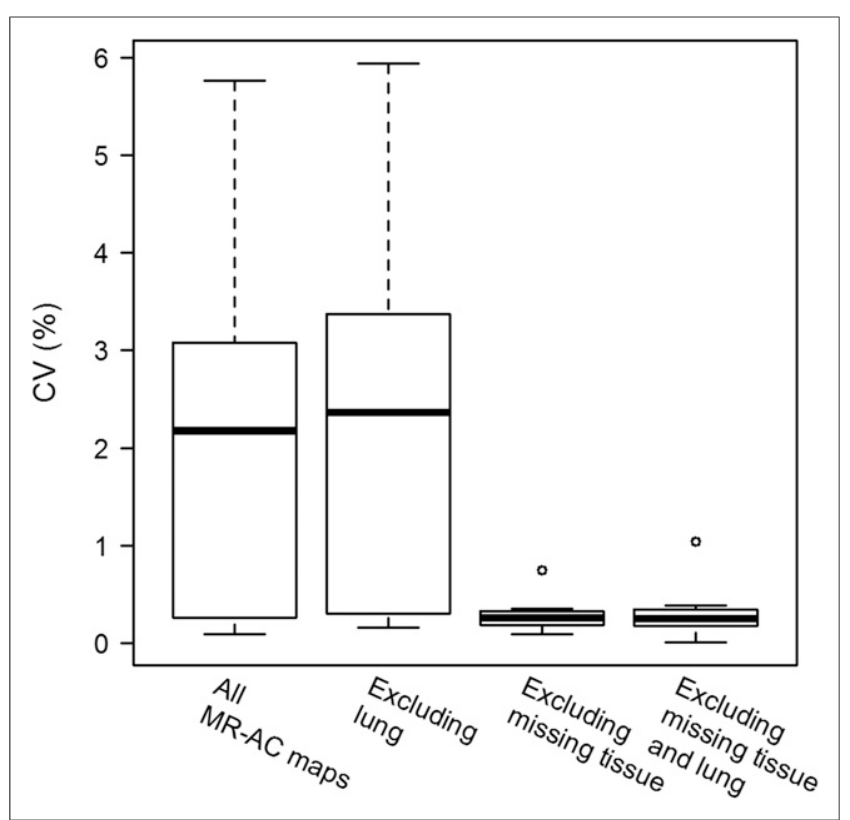

FIGURE 2. CV of total-body volume (TB). CVs are given for all MR-AC maps and for MR-AC maps excluding missing tissue artifacts, before and after exclusion of lung compartment.

lung, respectively (Fig. 2). The mean CVs of the subcompartment volumes relative to the total-body volume were $0.9 \pm 1.1 \%, 2.9 \pm$ $4.1 \%, 3.6 \pm 3.9 \%$, and $4.2 \pm 1.8 \%$ for ST, fat, IT, and lung, respectively. The exclusion of missing tissue and tissue swap artifacts resulted in reduced CVs of $0.7 \pm 0.7 \%, 1.3 \pm 1.0 \%, 1.3 \pm 0.7 \%$, and $3.1 \pm 1.8 \%$, respectively (Fig. 3).

\section{LBM}

Linear regression was performed while restricting the intercept to zero and revealed a scaling factor of 1.08 and 1.06 for all MRAC maps and MR_AC maps excluding missing tissue and tissue swap artifacts, respectively.

The value range of $\mathrm{LBM}_{\text {Formula }}$ and $\mathrm{LBM}_{\mathrm{MR}-\mathrm{AC} \text { (Scaled) was } 85 \%-}$ $106 \%$ and $94 \%-106 \%$ of the $\mathrm{LBM}_{\mathrm{ADB}}$ respectively. When tissue swap and missing tissue artifacts were excluded, the range of $\mathrm{LBM}_{\mathrm{MR}-\mathrm{AC} \text { (Scaled) }}$ increased to $100 \%-106 \%$ of $\mathrm{LBM}_{\mathrm{ADP}}$. The results for each subject are listed in Table 1. Repeated tissue swap artifacts in 2 subjects were corrected manually for 1 scan each to obtain a theoretic, tissue swap artifact-free MR-AC map. This correction implied the manual inversion of fat and ST values in the affected regions. These data were not included in the calculations of the correlation and the linear regression but are included in Table 1 and Figure 4.

Figure 4 shows $\mathrm{LBM}_{\mathrm{MR}-\mathrm{AC}}$ and $\mathrm{LBM}_{\text {Formula }}$ in comparison to $\mathrm{LBM}_{\mathrm{ADP}}$. Pearson correlation between $\mathrm{LBM}_{\mathrm{ADP}}$ and $\mathrm{LBM}_{\mathrm{MR}-\mathrm{AC}}$ was $r=0.989$ (95\% CI, 0.954-0.998, $P<0.001)$ and $r=0.992$ (95\% CI, 0.958-0.999, $P<0.001$ ) for all MR-AC maps before and after exclusion of scans with tissue swap and missing tissue artifacts, respectively. Between $\mathrm{LBM}_{\mathrm{ADP}}$ and $\mathrm{LBM}_{\text {Formula }}$, the correlation was $r=0.893$ (95\% CI, 0.601-0.975, $P<0.001)$ and $r=$ 0.914 (95\% CI, 0.587-0.985, $P<0.001$ ), respectively.

\section{DISCUSSION}

Combined PET/MR imaging mandates the use of MR-based image information to derive attenuation maps as a prerequisite to
PET quantification (24). This study had 2 objectives: first, to assess the reproducibility of standard MR-based attenuation maps using human subjects, and second, to investigate whether this Dixon-based approach to MR-AC could facilitate a straightforward estimation of the LBM in human subjects, so as to provide an intrinsic means of performing SUV calculations based on the use of LBM (12).

We were able to demonstrate the limitations of reproducibility of MR-AC using standard, Dixon-based imaging methods (Table 2; Fig. 1). Above all, image artifacts in MR-AC were rather frequent in this study. As expected from the standard implementation of the Dixon approach to calculate the MR-AC in the Biograph mMR, truncation artifacts were observed in all subjects. During the development of PET/CT, this issue was also a problem, but it could be solved by implementing dedicated procedures for expanding the field of view (25). Similar promising and effective procedures have already been proposed and validated for PET/MR imaging and can be used to overcome this problem $(26,27)$.

Tissue swap artifacts were observed also, albeit in fewer subjects. These artifacts are well known in Dixon-based MR-AC and most prominent in lean patients $(8,10)$. Nevertheless, solutions to correcting for tissue swaps have already been described, for example, by Wald et al., who identified tissue swaps in lower extremities using difference images of overlapping slices of contiguous bed positions (10). Switching the information of the interchanged sequence images is used to correct for these distortions in a repeated scan.

In our cohort the observed missing tissue artifacts were always located in the axial region covered by the last surface coil, that is, in the area of the knees (Fig. 1). For imaging the remainder of the lower extremities, the internal MR body coil was used because of the limited acceptable number of surface coils in parallel operation. In our experience, these missing tissue artifacts are frequently caused by an incorrect automatic selection of the optimal receiving coil near the transition zone between add-on surface

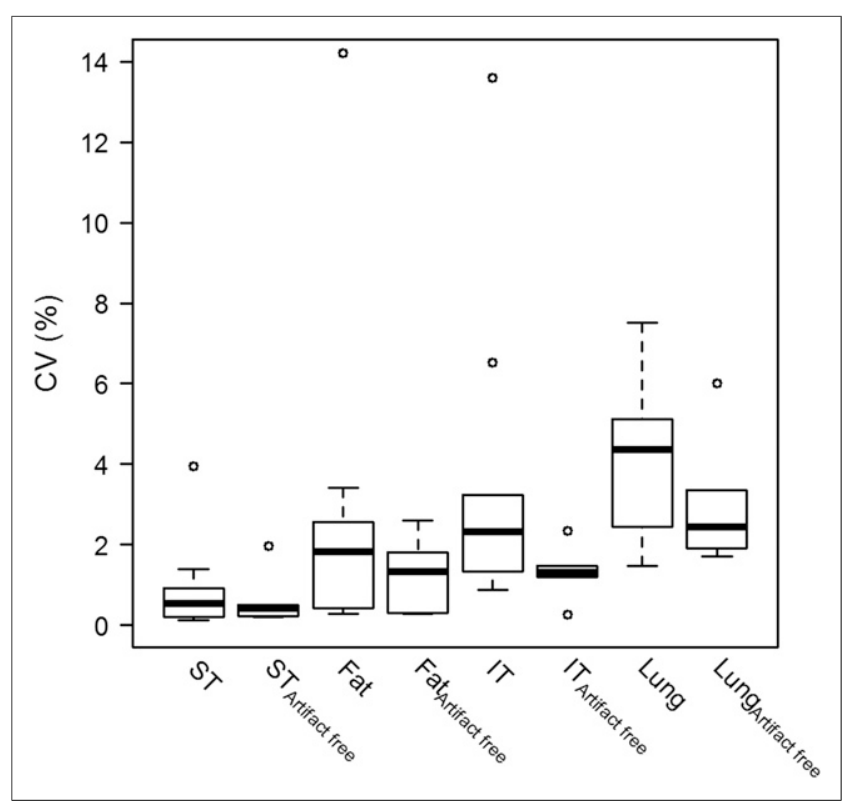

FIGURE 3. CV of subcompartment volumes. CVs are shown for all MR-AC maps and for MR-AC maps excluding tissue swap and missing tissue artifacts (i.e., artifact-free). 


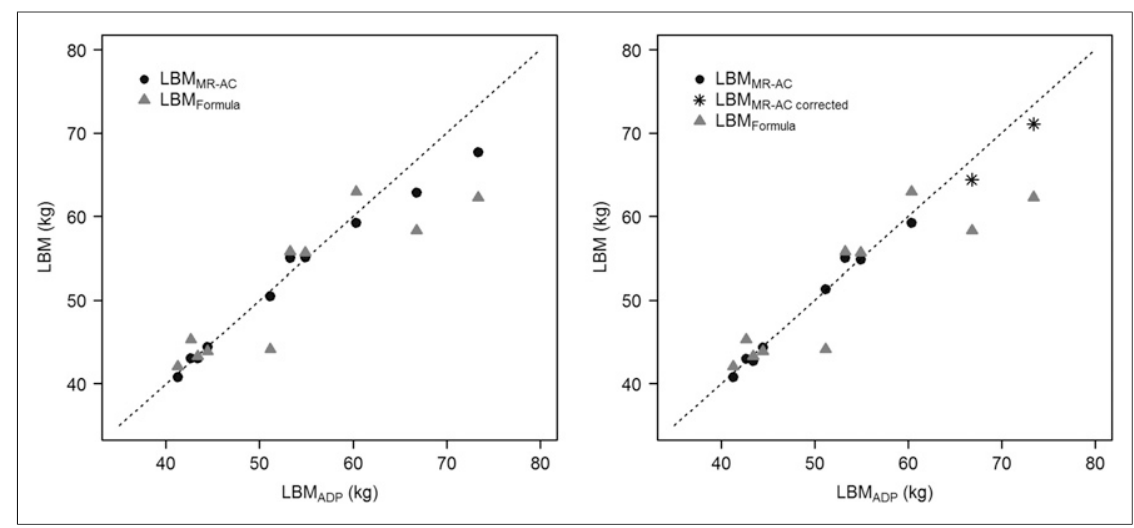

FIGURE 4. Comparison of scaled LBM $\mathrm{MR}_{\mathrm{AC}}$ and $\mathrm{LBM}_{\mathrm{Formula}}$ with $\mathrm{LBM}_{\mathrm{ADP}}$ : using all MR-AC acquisitions (left) and excluding tissue swap and missing tissue artifacts (right). LBM MR-AC corrected values $\left(^{*}\right)$ are LBM $_{\text {MR-AC }}$ for the 2 volunteers with tissue swaps in all acquisitions for a single acquisition in which tissue swaps have been manually corrected.

coils and the intrinsic body coil. Therefore, missing tissue artifacts are likely to disappear in examinations when the axial field of view is covered completely with surface coils (e.g., a standard whole-body examination) or when only the integrated body coils are used for the entire acquisition. Moreover, a manual selection of the receiving coils in the affected region can also help to overcome this artifact.

Air cavities occurred in regions in which air can be expected (e.g., abdominal region, air cavities in head). However, these cavities appeared to be overestimated in size because of susceptibility effects. This is an inherent challenge to MRI arising from magnetic field inhomogeneities at the interface of air and tissue $(7,28)$.

The reproducibility of total-body volume is limited (CV, 2.1\%) for all subjects and test-retest scans. However, given several a priori solutions for many of these image distortions, as outlined above, we estimated this reproducibility also in the absence of the most critical image distortions (missing tissue), only to find a significantly reduced $\mathrm{CV}$ of $0.3 \%$. This variance is similar to that of standard MR images and in accordance with the literature. For example, Wald et al. calculated a CV of $0.3 \%$ for total-body volume assessed in Dixon-based fat quantification (10).

Further, we estimated the effect of the observed variance of the MR-AC maps on the estimated PET activity values, thereby acknowledging the fact that no PET tracer was injected to the subjects of this study. For example, for a subject of $80-\mathrm{kg}$ weight and $180-\mathrm{cm}$ height with an estimated body volume of about $80 \mathrm{~L}$ (assuming a density of about $1 \mathrm{~kg} / \mathrm{L}$ ) and a body surface area of $2 \mathrm{~m}^{2}$ (29), a reported CV of $0.3 \%$ would result in an SD of the change in volume of $240 \mathrm{~mL}$. If this difference in volume was to be evenly distributed across the entire body, then it could be represented by an additional layer tissue of $0.12 \mathrm{~mm}$ across the entire body surface. Therefore, an annihilation photon pair originating from the center of the body in the transaxial direction would face an additional layer of attenuating material of approximately $0.24 \mathrm{~mm}$; such increase in relative layer thickness, however, is insignificant in view of the entire attenuation path across the body and, therefore, is not likely to cause noticeable differences in attenuation-corrected PET data.

Subcompartment volumes showed a slightly lower stability (CV, $0.7 \%-1.3 \%$ ), with the exception of the lung compartment $(\mathrm{CV}, 3.1 \%)$. This is expected because the phase of respiration may change between acquisitions. Compared with the literature, the reproducibility was in line with MR-based body composition measurements $(10,11,30)$. For example, Ludwig et al. calculated a $1.3 \%$ maximum $\mathrm{CV}$ for the fat compartment extracted automatically from Dixon-based MR images for 5 repeated scans (11), which compares well with the results of our study (CV, 1.3\%). Likewise, these changes should not have a noticeable impact on attenuation-corrected PET data.

In summary, the stability of the Dixonbased MR-AC is comparable to advanced body composition measurements using MRI, indicating the usability of MR-AC for LBM assessment. Therefore, we compared LBM $_{\text {MR-AC }}$ with LBM estimated with ADP and a predictive formula. ADP is a well-established method for body composition assessment, and several studies set the range of $\mathrm{CV}$ for this method to between $1.7 \%$ and $3.7 \%$ (19). In this study, the correlation of $\mathrm{LBM}_{\mathrm{ADP}}$ and $\mathrm{LBM}_{\mathrm{MR}-\mathrm{AC}}$ was excellent $(r=0.99)$. A lower correlation was found between $\mathrm{LBM}_{\mathrm{ADP}}$ and $\mathrm{LBM}_{\text {Formula }}$ $(r=0.89)$, which can be explained by the limited performance of the predictive formulas in nonstandard patients, such as in very lean (Table 1) or obese subjects (23). In these atypical subjects, $\mathrm{LBM}_{\mathrm{MR}-\mathrm{AC}}$ is expected to be more accurate than $\mathrm{LBM}_{\text {Formula }}$.

Nevertheless, $\mathrm{LBM}_{\mathrm{MR}-\mathrm{AC}}$ was underestimated in comparison to $\mathrm{LBM}_{\mathrm{ADP}}$. This systematic deviation is most likely caused by the properties of the MR-AC segmentation algorithm in combination with the model used for the LBM calculation. Similar deviations were reported for automated fat quantifications using Dixon images on MRI systems (11). More specifically, the authors found significant correlations (Pearson) of $r=0.98$ and 0.97 with ADP measurements for 2 different models to calculate the fat mass. However, depending on the model used, average deviations of the absolute values of $+18 \%$ and $-11 \%$ were reported (11).

In our study, a linear regression was used to obtain a calibration factor to correct for the systematic deviation of $\mathrm{LBM}_{\mathrm{MR}-\mathrm{AC}}$ and $\mathrm{LBM}_{\mathrm{ADP}}$. The intercept was restricted to zero, as an imaginary person containing no fat measured in both modalities should result in a zero fat fraction in both measurements.

The data presented in this study were obtained from total-body measurements. In clinical practice, the most prominent axial investigation area is from the base of the skull to mid thighs $(18,31)$, and therefore, image information of the head and the lower extremities are typically missing. Although these missing body parts are not expected to cause large errors in the LBM estimation (32), a follow-up investigation should target a predictive extrapolation of these regional volumes from the axial imaging range covered in a standard whole-body study.

\section{CONCLUSION}

The reproducibility of the Dixon-based MR-AC is high for artifact-free images and for images with only minor distortions. Nevertheless, in current implementation of MR-AC the frequency of artifacts is comparatively high, thus limiting the reproducibility of standard MR-AC. However, in the case of subjects void of tissue swap and missing tissue artifacts in MR-AC data, LBM 
estimation from Dixon-based MR-AC maps correlates well with standard LBM measurements and offers the option for routine $\mathrm{SUV}_{\mathrm{LBM}^{-}}$-based quantification in PET/MR.

\section{DISCLOSURE}

The costs of publication of this article were defrayed in part by the payment of page charges. Therefore, and solely to indicate this fact, this article is hereby marked "advertisement" in accordance with 18 USC section 1734. No potential conflict of interest relevant to this article was reported.

\section{ACKNOWLEDGMENTS}

We thank Ines Haidinger for her assistance with the acquisition of the MR images and Jacqueline Atanelov for her advice during the first test measurements. Furthermore, we are grateful to Matthias Fenchel for his contributions to establishing Dixon-based MR-AC in our study.

\section{REFERENCES}

1. Basu S, Kwee TC, Surti S, Akin EA, Yoo D, Alavi A. Fundamentals of PET and PET/CT imaging. Ann N Y Acad Sci. 2011;1228:1-18.

2. Kinahan PE, Townsend DW, Beyer T, Sashin D. Attenuation correction for a combined 3D PET/CT scanner. Med Phys. 1998;25:2046-2053.

3. Bailey DL, Antoch G, Bartenstein P, et al. Combined PET/MR: the real work has just started-summary report of the third international workshop on PET/MR imaging; February 17-21, 2014, Tuebingen, Germany. Mol Imaging Biol. 2015; 17:297-312.

4. Torigian DA, Zaidi H, Kwee TC, et al. PET/MR imaging: technical aspects and potential clinical applications. Radiology. 2013;267:26-44.

5. Keereman V, Mollet P, Berker Y, Schulz V, Vandenberghe S. Challenges and current methods for attenuation correction in PET/MR. MAGMA. 2013;26:81-98.

6. Bailey DL, Barthel H, Beuthin-Baumann B, et al. Combined PET/MR: where are we now? Summary report of the second international workshop on PET/MR imaging April 8-12, 2013, Tuebingen, Germany. Mol Imaging Biol. 2014;16: 295-310.

7. Keller SH, Holm S, Hansen AE, et al. Image artifacts from MR-based attenuation correction in clinical, whole-body PET/MRI. MAGMA. 2013;26:173-181.

8. Ladefoged CN, Hansen A, Keller S, et al. Impact of incorrect tissue classification in Dixon-based MR-AC: fat-water tissue inversion. EJNMMI Phys. 2014;1:101.

9. Lassen M, Quick H, Boellaard R, et al. Accuracy and reproducibility of standard MR-based attenuation maps of the thorax: an inter-system comparison of wholebody PET/MR systems [abstract]. Eur J Nucl Med Mol Imaging. 2014;41(suppl 2):S408.

10. Wald D, Teucher B, Dinkel J, et al. Automatic quantification of subcutaneous and visceral adipose tissue from whole-body magnetic resonance images suitable for large cohort studies. J Magn Reson Imaging. 2012;36:1421-1434.

11. Ludwig UA, Klausmann F, Baumann S, et al. Whole-body MRI-based fat quantification: a comparison to air displacement plethysmography. J Magn Reson Imaging. 2014;40:1437-1444.
12. Jochimsen TH, Schulz J, Busse H, et al. Lean body mass correction of standardized uptake value in simultaneous whole-body positron emission tomography and magnetic resonance imaging. Phys Med Biol. 2015;60:4651-4664.

13. Carson RE. Tracer kinetic modeling in PET. In: Bailey D, Townsend D, Valk P, Maisey M, eds. Positron Emission Tomography. London, U.K.: Springer-Verlag; 2005:127-159.

14. Thie JA. Understanding the standardized uptake value, its methods, and implications for usage. J Nucl Med. 2004;45:1431-1434.

15. Hoekstra CJ, Paglianiti I, Hoekstra OS, et al. Monitoring response to therapy in cancer using tomography: an overview of different analytical methods. Eur J Nucl Med. 2000;27:731-743.

16. Sugawara Y, Zasadny KR, Neuhoff AW, Wahl RL. Reevaluation of the standardized uptake value for FDG: variations with body weight and methods for correction. Radiology. 1999;213:521-525.

17. Raijmakers P, Temmerman OPP, Saridin CP, et al. Quantification of ${ }^{18} \mathrm{~F}$-fluoride kinetics: evaluation of simplified methods. J Nucl Med. 2014;55:1122-1127.

18. Rausch I, Bergmann H, Geist B, et al. Variation of system performance, quality control standards and adherence to international FDG-PET/CT imaging guidelines. Nuklearmedizin. 2014;53:242-248.

19. Fields DA, Goran MI, McCrory MA. Body-composition assessment via airdisplacement plethysmography in adults and children: a review. Am J Clin Nutr. 2002;75:453-467.

20. Dempster P, Aitkens S. A new air displacement method for the determination of human body composition. Med Sci Sports Exerc. 1995;27:1692-1697.

21. Martinez-Möller A, Souvatzoglou M, Delso G, et al. Tissue classification as a potential approach for attenuation correction in whole-body PET/MRI: evaluation with PET/CT data. J Nucl Med. 2009;50:520-526.

22. Coward WA, Parkinson SA, Murgatroyd PR. Body composition measurements for nutrition research. Nutr Res Rev. 1988;1:115-124.

23. Green B, Duffull SB. What is the best size descriptor to use for pharmacokinetic studies in the obese? Br J Clin Pharmacol. 2004;58:119-133.

24. Bezrukov I, Mantlik F, Schmidt H, Schölkopf B, Pichler BJ. MR-based PET attenuation correction for PET/MR imaging. Semin Nucl Med. 2013;43:45-59.

25. Beyer T, Bockisch A, Kühl H, Martinez M-J. Whole-body ${ }^{18}$ F-FDG PET/CT in the presence of truncation artifacts. J Nucl Med. 2006;47:91-99.

26. Schramm G, Langner J, Hofheinz F, et al. Influence and compensation of truncation artifacts in MR-based attenuation correction in PET/MR. IEEE Trans Med Imaging. 2013;32:2056-2063.

27. Blumhagen JO, Braun H, Ladebeck R, et al. Field of view extension and truncation correction for MR-based human attenuation correction in simultaneous MR/PET imaging. Med Phys. 2014;41:022303.

28. Dietrich O, Reiser MF, Schoenberg SO. Artifacts in 3-T MRI: physical background and reduction strategies. Eur J Radiol. 2008;65:29-35.

29. Haycock GB, Schwartz GJ, Wisotsky DH. Geometric method for measuring body surface area: a height-weight formula validated in infants, children, and adults. J Pediatr. 1978;93:62-66.

30. Machann J, Thamer C, Schnoedt B, et al. Standardized assessment of whole body adipose tissue topography by MRI. J Magn Reson Imaging. 2005;21: 455-462.

31. Beyer T, Czernin J, Freudenberg LS. Variations in clinical PET/CT operations: results of an international survey of active PET/CT users. J Nucl Med. 2011;52: 303-310.

32. Hamill JJ, Sunderland JJ, Leblanc AK, Kojima CJ, Wall J, Martin EB. Evaluation of CT-based lean-body SUV. Med Phys. 2013;40:092504. 